\title{
Genetic variants in Chinese patients with sporadic dilated cardiomyopathy: a cross-sectional study
}

\author{
Cheng Shen ${ }^{1,2}$, Lei $\mathrm{Xu}^{1}$, Xiaoning $\mathrm{Sun}^{3}$, Aijun Sun ${ }^{1,4}$, Junbo Ge $\mathrm{G}^{1,4}$ \\ ${ }^{1}$ Department of Cardiology, Zhongshan Hospital, Fudan University, Shanghai Institute of Cardiovascular Diseases, Shanghai, China; ${ }^{2}$ Department of \\ Cardiology, Affiliated Hospital of Jining Medical University, Jining Key Laboratory for Diagnosis and Treatment of Cardiovascular Diseases, Jining, \\ China; ${ }^{3}$ Department of Cardiovascular Surgery, Zhongshan Hospital, Fudan University, Shanghai Institute of Cardiovascular Diseases, Shanghai, \\ China; ${ }^{4}$ Institutes of Biomedical Sciences, Fudan University, Shanghai, China \\ Contributions: (I) Conception and design: L Xu, X Sun; (II) Administrative support: A Sun, J Ge; (III) Provision of study materials or patients: C \\ Shen, L Xu, X Sun; (IV) Collection and assembly of data: C Shen, L Xu; (V) Data analysis and interpretation: C Shen, L Xu, X Sun; (VI) Manuscript \\ writing: All authors; (VII) Final approval of manuscript: All authors. \\ Correspondence to: Dr. Lei Xu; Dr. Xiaoning Sun. Fenglin Road 180, Shanghai, China. Email: bri3stone@163.com; sun.xiaoning@zs-hospital.sh.cn.
}

Background: Multiple genes have been associated with familial dilated cardiomyopathy (DCM). However, the role of genetic factors in sporadic DCM (SDCM) remains unclear. Therefore, we studied the genetic variations in Chinese patients with SDCM.

Methods: Sixty-six unrelated Chinese patients (mean age $49.1 \pm 17.0$ years; $71 \%$ male) diagnosed with SDCM were enrolled. The clinical history and genomic DNA of the cohort were collected and examined. The exons of 24 genes closely associated with familial DCM (ABCC9, ACTC1, ACTN2, DES, LAMA4, LDB3, LMNA, MYBPC3, MYH6, MYH7, MYPN, PLN, PSEN1, PSEN2, RBM20, SCN5A, SGCD, TAZ, TCAP, TMPO, TNNI3, TNNT2, TPM1, and $V C L$ ) were sequenced using targeted next-generation sequencing method. All called nonsynonymous variants and their occurrence frequencies were compared against population data from public databases. And the nonsynonymous variants were also evaluated for pathogenicity by PolyPhen 2 (PP2) and Sorts Intolerant From Tolerant (SIFT) algorithms.

Results: Eighty-five nonsynonymous variants were detected in 17 genes. The variants and their occurrence frequencies in the patients were compared against population data from the 1000 Genomes and NHLBI (National Heart, Lung, and Blood Institute) Go Exome Sequencing Project. Forty-nine nonsynonymous variants had occurrence frequencies that were significantly higher in the study patients than in the general population, indicating that they have the potential to increase the risk of DCM. The risk variants were distributed in $40(61 \%)$ patients, among whom 25 carried a single variant, while the remaining patients carried multiple (2 to 4) variants. Risk variants occurred more frequently in $M Y B P C 3$ (14\% of the patients), SCN5A (14\%), MYH7 (12\%), MYPN (9\%), and LDB3 (8\%), as verified by Poisson distribution analysis, which were considered "the five risky genes".

Conclusions: We found that genetic variants with potential risk for DCM were commonly present in SDCM patients, indicating that genetic factors contribute to the pathogenesis, and (probably) the onset, of DCM in these patients.

Keywords: Sporadic dilated cardiomyopathy (SDCM); mutations; risky genes

Submitted Nov 25, 2021. Accepted for publication Jan 05, 2022.

doi: 10.21037/atm-21-6774

View this article at: https://dx.doi.org/10.21037/atm-21-6774 


\section{Introduction}

Dilated cardiomyopathy (DCM) is characterized by left ventricular (LV) dilatation and impaired systolic function, and is a leading cause of heart failure (1). Idiopathic DCM is defined when no other discernible causes, such as ischemia, valvular disease, and myocarditis, are present. Epidemiological studies have shown that a proportion of idiopathic DCM cases are familial (2). Familial DCM (FDCM) is primarily transmitted through an autosomal dominant pattern, while other patterns, including $\mathrm{X}$-chromosomal, autosomal recessive, and mitochondrial transmission, are much less common or rare. To date, an increasing number of individual genes have been associated with inheritance in familial DCM cases $(1,2)$.

However, more idiopathic DCM cases are sporadic, and the etiology of sporadic DCM (SDCM) remains largely unknown. SDCM patients are diagnosed with DCM manifestation but no family history, which are common in clinical practice. So far, compared to the information obtained from a large number of studies on FDCM cases, much less information is available regarding the role of genetic factors, such as rare genetic variations, on the pathogenesis of SDCM. Previously, a few studies on mutations of several individual genes implicated the role of genetic factors in the development of SDCM (3-6). Several cohorts, mainly containing Caucasian patients with FDCM or a combination of FDCM and SDCM, were also investigated for mutations in a relatively small number of selected candidate genes, including $M Y H 7$, TNNT2, SCN5A, CSRP3, and LBD (7-9).The consideration of genetic variants is important when evaluating the pathogenicity of a genomic variant. The genetic variants were identified using the genome analysis toolkit and the genetic databases. A systematic assessment of correlation between more candidate genes and SDCM cases would help to determine whether SDCM has genetic influences, and if so, whether SDCM shares the same or similar sets of genetic risk factors that are associated with FDCM, and hence, whether these two forms of DCM share similar or major pathogenetic paths. Li et al. reported some genetic variants in 24 SDCM patients recently, while the sample size is small (10). We aimed to expand the subjective scale and study the characteristics of genetic variants in 66 unrelated Chinese patients with SDCM using a nextgeneration sequencing technique targeting on 24 genes that have been previously reported to be associated with DCM primarily based on familial cases $(11,12)$. We present the following article in accordance with the STROBE reporting checklist (available at https://atm.amegroups.com/article/ view/10.21037/atm-21-6774/rc).

\section{Methods}

\section{Subjects}

All procedures performed in this study involving human participants were in accordance with the Declaration of Helsinki (as revised in 2013). This cross-sectional clinical investigation was conducted in compliance with the guidelines for genetic research in the protocol approved by the Ethics Committees of Zhongshan Hospital (No. 2006-87). All participants signed a written informed consent. All participants in this study were unrelated individuals who were Han Chinese living in eastern China. Patients diagnosed with idiopathic DCM were hospitalized and recruited into the study from the Cardiology Department of Zhongshan Hospital. The inclusion criteria for the SDCM cohort were consistent with the guidelines described by the AHA Scientific Statement and position statement of the ESC working group $(13,14)$. The exclusion criteria included peripartum cardiomyopathy and secondary dilated cardiomyopathies caused by ischaemic heart diseases, hypertension, valvular diseases, endocrine disorders (such as diabetic cardiomyopathy and hyperthyroid cardiomyopathy), inflammation, toxicity, and stress. All patients in this study reported no family history of DCM based on their recollection. Further clinical and genetic screenings were not conducted.

\section{Clinical data collection}

Clinical information available from the study subjects included date of birth, gender, vital status, clinical diagnosis, age at diagnosis (age at genetic testing was recorded if the age at diagnosis was not available), family history of DCM and other cardiovascular or muscular diseases, cardiovascular history (including myocardial infarction, hypertension, myocarditis, and drug/toxin exposure), and cardiac structure and function [including maximal $\mathrm{LV}$ wall thickness, LV ejection fraction (LVEF), and LV dimensions].

\section{Genomic DNA (deoxyribonucleic acid) sequencing and data analysis}

Genomic DNA was isolated from the peripheral blood of the participants. From each sample, $5 \mu \mathrm{g}$ of genomic DNA was dissolved in $50 \mu \mathrm{L}$ of water and fragmented to a size 
of 100-300 bp, as judged by agarose gel electrophoresis. The patients' DNA samples were screened using a nextgeneration sequencing technique for point mutation variants in exons of the 24 genes that were reported to be associated with DCM primarily based on FDCM cases. The following genes were included: $A B C C$ 9 (ATP-sensitive potassium channel regulatory subunit SUR2A), ACTC1 (cardiac actin), ACTN2 ( $\alpha$-actinin-2), DES (desmin), LAMA4 (laminin a-4), LDB3 (cypher), LMNA (lamin A/C), MYBPC3 (myosinbinding protein $\mathrm{C}$ ), MYH6 ( $\alpha$-myosin heavy chain), MYH7 ( $\beta$-myosin heavy chain), MYPN (myopalladin), PLN (phospholamban), PSEN1 (presenilin 1), PSEN2 (presenilin 2), RBM20 (RNA binding protein 20), SCN5A (cardiac sodium channel), $S G C D$ ( $\delta$-sarcoglycan), TAZ (tafazzin), TCAP (telethonin), TMPO (thymopoietin), TNNI3 (cardiac troponin I), TNNT2 (cardiac troponin T), TPM1 (tropomyosin $\alpha-1$ chain), and VCL (metavinculin). Next-generation sequencing was performed at the sequencing platform of the Shanghai Institute of Cardiovascular Diseases.

Variants were called by aligning the raw sequence data to the human GRCh37 reference genome with manual verification. Novel variants were defined as those variants that were not found in the SNP Database (dbSNP) build 137 of the National Center for Biotechnology Information, and that had not been reported at the completion of the study. All called nonsynonymous variants of the genes in the DCM cohort and their occurrence frequencies were compared against population data from public databases, including the 1000 Genomes Project (http:// www.1000genomes.org), and NHLBI (National Heart, Lung, and Blood Institute) Go Exome Sequencing Project (http://evs.gs.washington.edu/EVS/). The sequencing data of the 197 Chinese adults deposited in the 1000 Genomes Project database were used as the Chinese (ethnic-matched) control group in this study. Sequence and occurrence frequency data from other populations in this database, and those from African American and European American populations in the NHLBI Go Exome Sequencing Project database were combined together and used as the control group of the non-Chinese populations.

The relationship between the nonsynonymous variants and DCM was evaluated by comparing the occurrence frequencies of the DCM cohort and the control populations. Details of the comparison are described in the Results section below. The nonsynonymous variants were also evaluated for pathogenicity by PolyPhen 2 (PP2) and Sorts Intolerant From Tolerant (SIFT) algorithms. PP2 scores were obtained using HumVar model software (http://genetics.bwh.harvard.edu/pph2/index.shtml). PP2 scores ranged from 0 to 1 , with three levels of pathogenic potential, i.e., probably damaging, possibly damaging, and benign). SIFT scores were also obtained using a software tool (http://siftdna.org/www/Extended_SIFT_chr_coords_ submit.html). The SIFT scores range from 0 to 1 , and were divided into damaging or tolerated levels with 0.05 as the threshold value.

\section{Statistical analysis}

Normally distributed continuous data were presented as mean \pm standard deviation (SD) and compared using the Student $t$-test. However, if the normality test (ShapiroWilk) and/or equal variance test failed, the Mann-Whitney rank-sum test was used to compare the continuous data. Categorical variables were presented as frequencies and analyzed by Fisher's exact test using the Simple Interactive Statistical Analysis web-based software (SISA Binomial, Southampton, UK). In all statistical tests, $\mathrm{P}<0.05$ was used to determine whether differences were statistically significant. To evaluate the occurrence frequencies of genetic variants, a model of simple Poisson process was used. We assumed that the number of variants occurring in a given gene approximately follows the Poisson distribution, $\mathrm{p}(\mathrm{k})=\mathrm{e}^{-\lambda} \lambda^{k} / k$ !. $\mathrm{P}<0.05$ indicated a significantly high occurrence frequency. Poisson distribution and $\mathrm{P}$ values were computed using a web-based tool (http://www. vassarstats.net/poissonfit.html\#down). The same method was also used to evaluate the significance of the prevalence of genetic variants.

\section{Results}

\section{Study cohort}

The SDCM cohort included 66 patients (47 males). The average age at diagnosis of patients in this cohort was $49.1 \pm 17.0$ years. All enrolled patients had symptoms of heart failure at the time of enrollment, with a majority of them in the New York Heart Association's (NYHA) classes III and IV. Echocardiographic findings showed a mean LV end-diastolic diameter (LVEDD) of $69 \pm 9 \mathrm{~mm}$, a mean $\mathrm{LV}$ end-systolic diameter (LVESD) of $57 \pm 10 \mathrm{~mm}$, and a mean LVEF of $34 \% \pm 10 \%$. 


\section{Nonsynonymous variants of DCM-associated genes in the SDCM cobort}

A total of 85 nonsynonymous variants, mostly singlenucleotide mutations, were detected by sequencing in 17 out of the 24 DCM-related genes in the DCM cohort (listed in Table 1). In this cohort, nonsynonymous variants were absent in seven DCM-related genes. Fifty-five (65\%) of the called nonsynonymous variants were either registered in the databases we searched or were reported previously (we denoted these variants as 'known variants'). The remaining $30(35 \%)$ of the called nonsynonymous variants have not yet been recorded anywhere else, and were therefore denoted herein as 'novel variants'. All novel variants were rare frequency mutations (i.e., allele frequency $<0.5 \%$ in the combined control populations). Meanwhile, among the 55 known variants, 19 (35\%) had higher allele frequencies $(>0.5 \%)$ in the combined control populations.

\section{Occurrence frequencies of the nonsynonymous variants}

To determine the possible relationship between the nonsynonymous variants and DCM in SDCM patients, we first compared the occurrence frequency (allele frequency) of the variants identified in the DCM cohort of this study with those of the control populations. We used two control populations, i.e., Chinese and non-Chinese controls, as described in the Methods section above. In addition, we also combined the Chinese and non-Chinese controls to form the general control population. The comparison indicated that the variants could be divided into three groups with statistically separable patterns.

The allele frequencies of variants in the first pattern group in the SDCM cohort were significantly higher than those in the general control population, but were statistically similar between the Chinese and non-Chinese control populations. These statistical features suggest that the variants falling in this pattern group exhibit a potential risk for SDCM (herein, we denoted these variants as 'risk variants'). There were 49 variants (58\% of the total nonsynonymous variants called) in this risky group. Most risk variants were not present in the control populations, except for six variants that had very low allele frequencies in the control populations. All risk variants were heterozygous mutations, except for a patient who carried a homozygous E334K mutation. It is noteworthy that all 30 novel variants were risk variants.

The allele frequencies of the variants in the second pattern group were statistically different between the Chinese and non-Chinese populations, but were statistically similar between the SDCM group and the Chinese control group. These results suggest that variants with these statistical features (30 variants, $35 \%$ of the total variants called) exhibit specific occurrence in the Chinese population, but are not likely to indicate an increased risk of SDCM. We found that the allele frequencies of variants that did not fall in the previous two pattern groups in the SDCM cohort were statistically similar to those in the general control population, indicating that these six variants ( $7 \%$ of the total variants called) were shared globally and do not exhibit a specific risk of SDCM. We denoted variants in the second and third groups as 'low-risk variants'. Table 1 displays the statistical data that are summarized here.

The risk variants were distributed in 16 DCM-associated genes, which accounted for $94 \%$ of the genes on which nonsynonymous variants were found, and $67 \%$ of the total number of genes in our study. They were distributed in 40 (61\%) patients, among whom 25 carried a single variant, 11 carried two variants, two carried three variants, and one carried four variants. As shown in Table 2, the prevalence of risk variants of each gene ranged from 1 to 9 among the 66 patients. MYBPC3 and SCN5A exhibited the highest prevalence $(9 / 66,14 \%)$. Poisson distribution analysis revealed that $M Y B P C 3, S C N 5 A, M Y H 7, M Y P N$, and $L D B 3$ had significantly higher prevalence (14\%, 14\%, 12\%, 9\%, and $8 \%$, respectively) than the other genes. They had a combined prevalence of 31/66 (47\%) in the SDCM cohort when multiple risk variants of these genes in a patient were counted as a single occurrence. MYBPC3, SCN5A, MYH7, and $L D B 3$ also housed more risk variants than the other genes, and the Poisson distribution analysis identified that they had a significantly high probability to house risk variants. These results suggested that these genes (which we denoted as 'risky genes') were closely related to SDCM.

\section{Pathogenic potential of the nonsynonymous variants}

Seventeen known variants, 11 risky variants, and six low-risk variants have been previously found to increase susceptibility to some cardiac diseases, including Brugada syndrome, LV noncompaction, hypertrophic cardiomyopathy, and distal myopathy (Table 1). However, none of the variants were directly related to DCM, except for A1180V and R1193Q of $S C N 5 A$, which were reported in the DCM cases $(26,30)$.

To obtain further information on the pathogenic potential of the nonsynonymous variants of the DCM- 


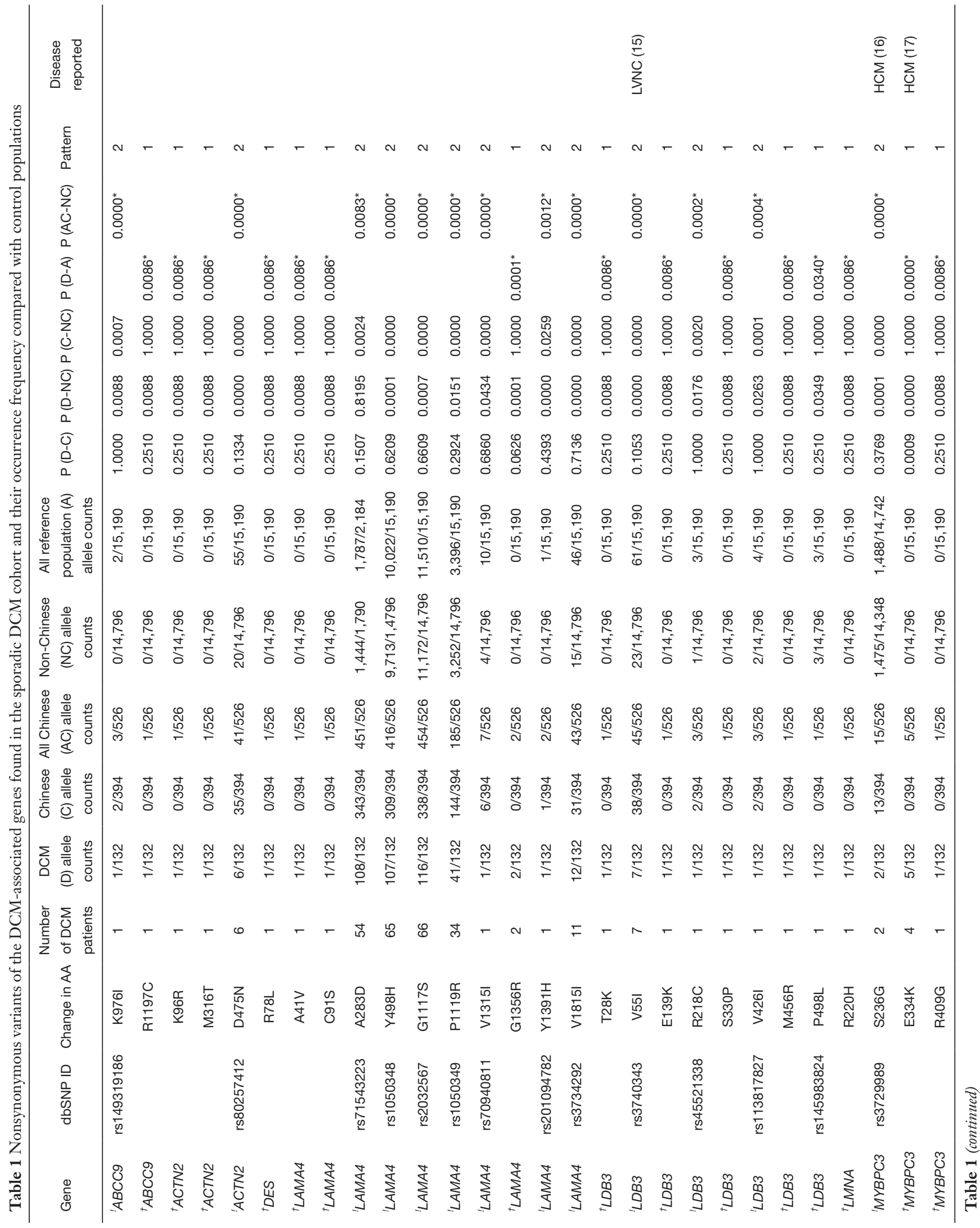




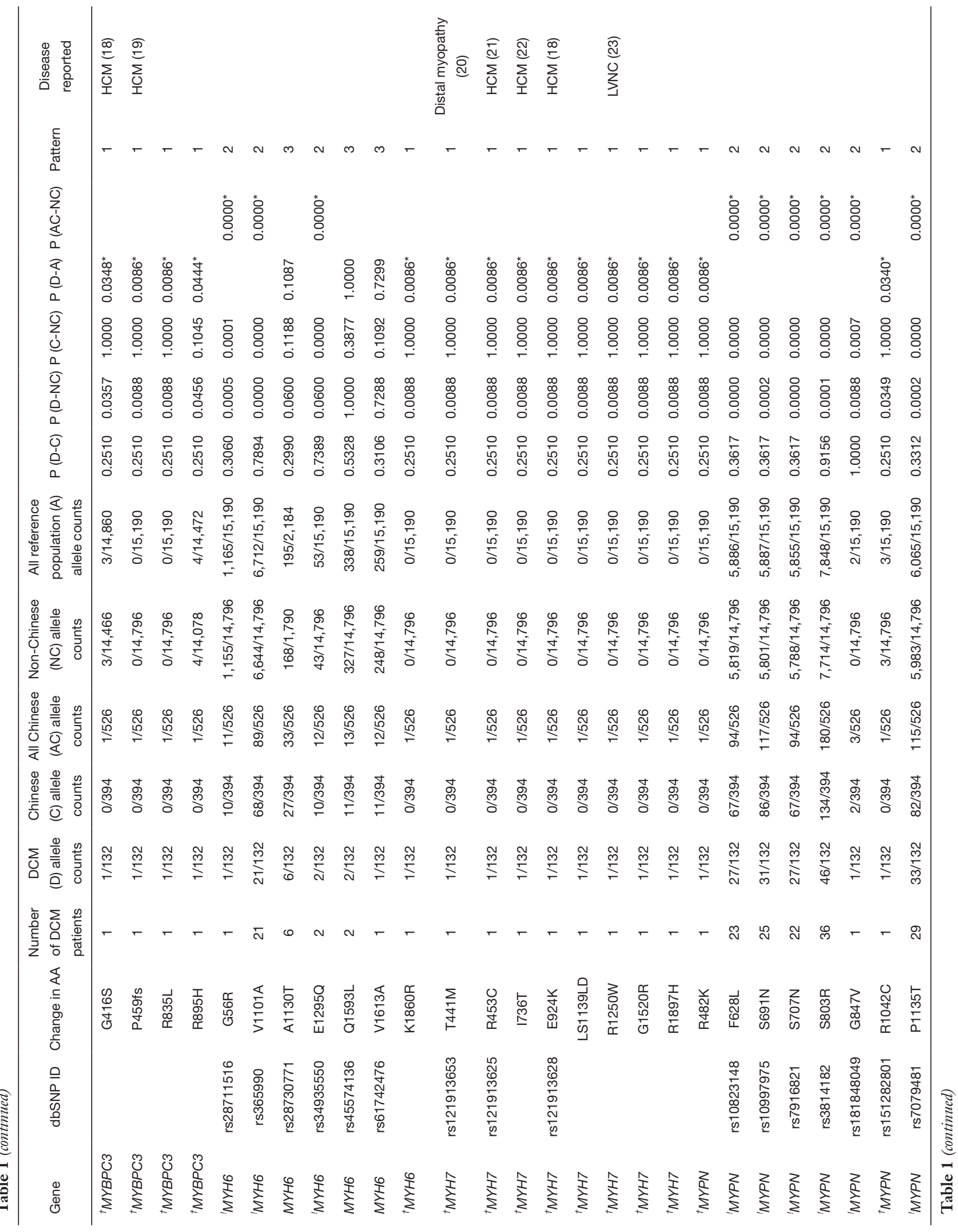




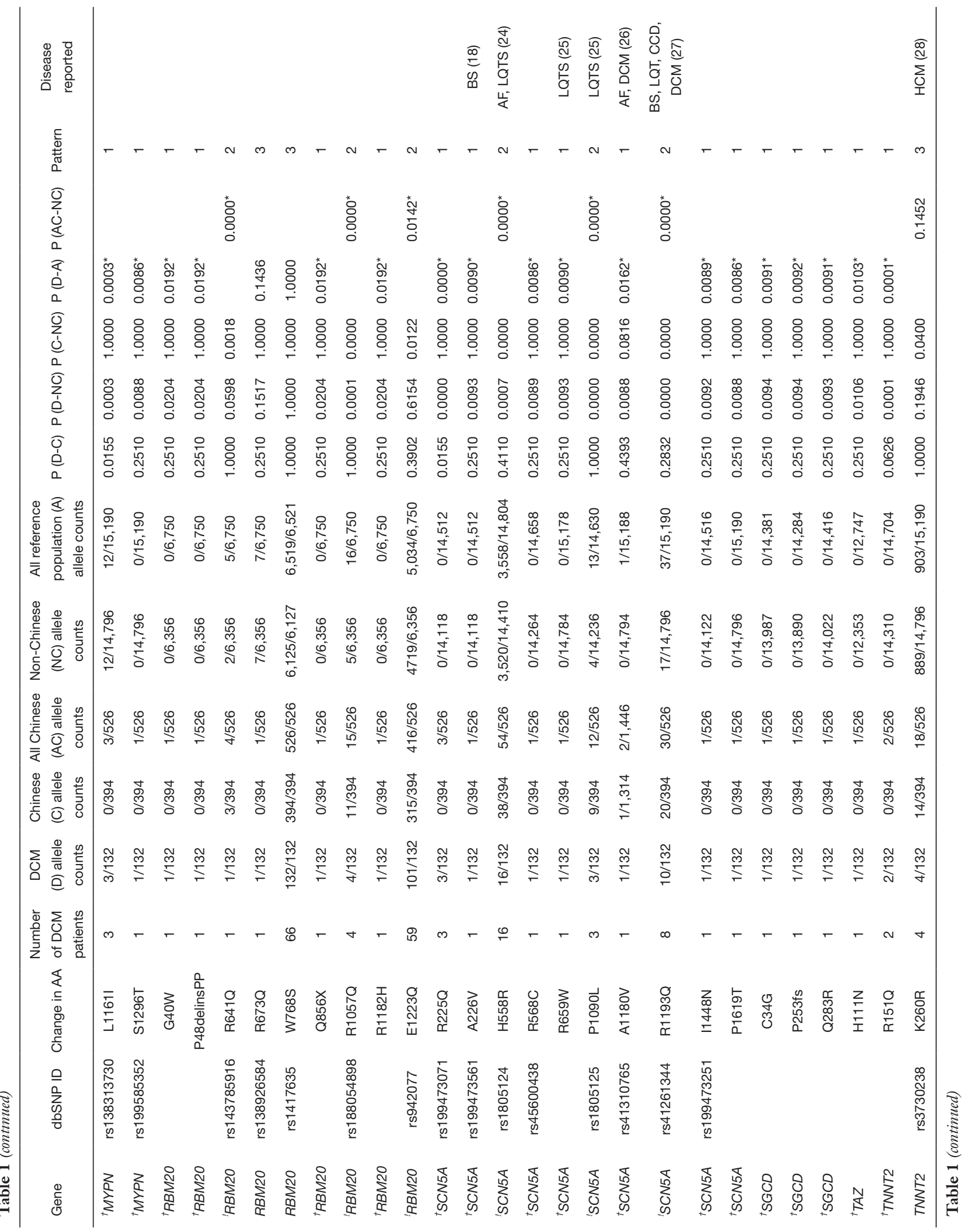




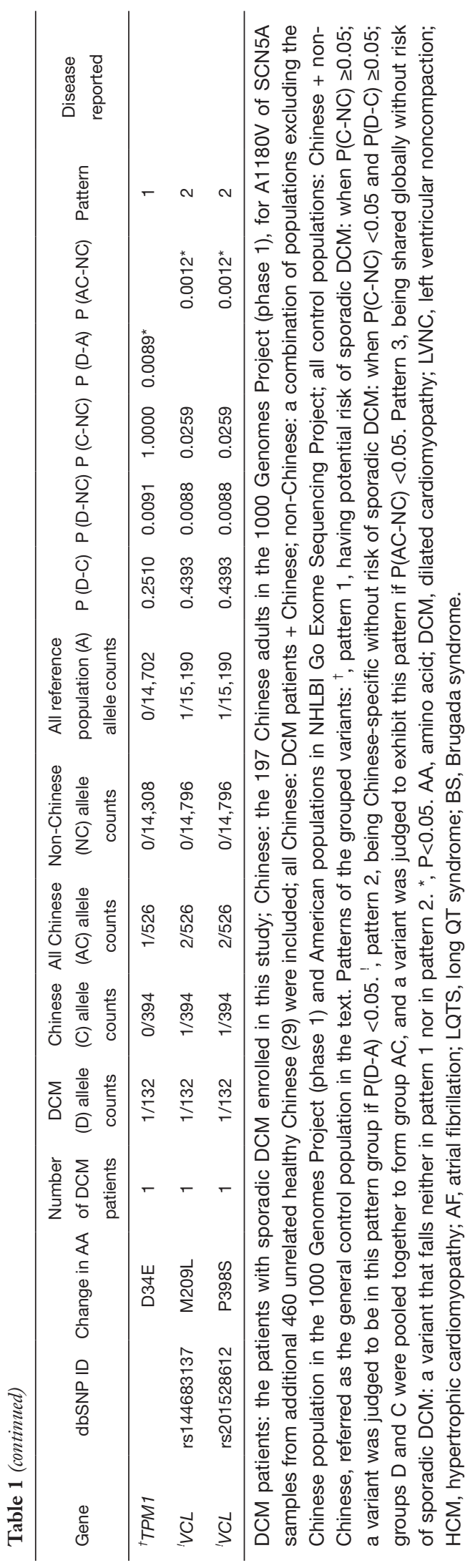

associated genes in the SDCM cohort, we performed an analysis of the nonsynonymous variants using the protein function prediction algorithms, PP2 and SIFT (Table S1). In total, 62 variants could be predicted by both algorithms ( $73 \%$ of the total nonsynonymous variants), which also included one non-sense and two frame-shift variants that could be assumed to cause damaging consequences in the protein function (31). Twenty-six risk variants $(53 \%$ of the total risk variants) were predicted to be damaging, and eight risk variants (16\% of the total risk variants) were predicted to be tolerated. On the other hand, 23 low-risk variants ( $63 \%$ of the total low-risk variants) were predicted to be tolerated, and five low-risk variants (14\% of the total low-risk variants) were predicted to be damaging. Fisher's exact test indicated a significant difference $(\mathrm{P}=0.000)$ in the predicted results between the risky and non-risky variant groups. Therefore, the protein function prediction algorithms confirmed that majority of the risk variants exhibit pathogenic potential, whereas the majority of lowrisk variants were predicted to be harmless based on the protein structure-function relationship. We noticed that in the risky genes of SDCM, SCN SA housed the most risk variants that were predicted to be damaging.

\section{Comparison of clinical symptoms between the patients with and without risk variants}

Given the determination of the risk variants, we further explored whether there were differences in clinical symptoms between the patients with and without risk variants, and compared the symptoms between these two groups of patients (as shown in Tables S2,S3). The results indicated that patients with risk variants did not manifest any symptoms or abnormalities that were significantly different from those seen in the patients without risk variants.

\section{Discussion}

This study was specifically designed to screen genes known to be associated with FDCM in SDCM patients. To our knowledge, similar studies have not yet been reported in the literature. In this study, we investigated a cohort of 66 unrelated Chinese patients with diagnosed SDCM. We performed mutational screening of 24 genes known to be associated with FDCM using a next-generation sequencing technique. A major finding of this study was that the atrisk genotypes are common $(61 \%)$ in SDCM patients. The 
Table 2 Genes hosting variants with potential risk of sporadic DCM in the DCM cohort being studied

\begin{tabular}{|c|c|c|c|c|c|c|}
\hline Gene & $\begin{array}{l}\text { Prevalence }{ }^{a} \text { of variants with } \\
\text { risk of sporadic DCM }\end{array}$ & $P$ value ${ }^{c}$ & $\begin{array}{l}\text { Prevalence }^{a} \text { of } \\
\text { variants }\end{array}$ & $\begin{array}{l}\text { Number of variants with } \\
\text { risk of sporadic DCM }\end{array}$ & $P$ value ${ }^{d}$ & Number of total variants \\
\hline МYBPCЗ & $9(13.6 \%)^{b}$ & $0.000^{*}$ & 11 & 6 & $0.010^{*}$ & 7 \\
\hline MYH7 & $8(12.1 \%)$ & $0.001^{*}$ & 8 & 8 & $0.001^{*}$ & 8 \\
\hline RBM20 & $4(6.1 \%)$ & 0.114 & 135 & 4 & 0.140 & 9 \\
\hline LAMA4 & $4(6.1 \%)$ & 0.114 & 236 & 3 & 0.261 & 10 \\
\hline$S G C D$ & $3(4.5 \%)$ & 0.277 & 3 & 3 & 0.261 & 3 \\
\hline$A B C C 9$ & $1(1.5 \%)$ & 0.840 & 2 & 1 & 0.830 & 7 \\
\hline$D E S$ & $1(1.5 \%)$ & 0.840 & 1 & 1 & 0.830 & 1 \\
\hline LMNA & $1(1.5 \%)$ & 0.840 & 1 & 1 & 0.830 & 1 \\
\hline TPM1 & $1(1.5 \%)$ & 0.840 & 1 & 1 & 0.830 & 1 \\
\hline$T A Z$ & $1(1.5 \%)$ & 0.840 & 1 & 1 & 0.830 & 1 \\
\hline$V C L$ & $0(0 \%)$ & 1.000 & 2 & 0 & 1.000 & 2 \\
\hline ACTC1 & $0(0 \%)$ & & 0 & 0 & & 0 \\
\hline TNNI3 & $0(0 \%)$ & & 0 & 0 & & 0 \\
\hline
\end{tabular}

a, prevalence was designated as the sum of patients carrying a variant (either homozygous or heterozygous) in each gene. When prevalence was calculated, variants co-occurring in a gene were counted separately. Likewise, variants co-occurring in a patient were also counted separately. ${ }^{b}$, prevalence in this column was also expressed as a percentage of the total patients in the DCM cohort. ${ }^{c, d}$, P values were computed using Poisson distribution as described in the Methods section. The means of the fitted Poisson distribution ( $\lambda$ ) were 0.97 and 0.95 , respectively. Only those genes that contained nonsynonymous variants were included in the computation. ${ }^{*}, \mathrm{P}<0.05$.

second major finding was that $M Y B P C 3$ and $S C N 5 A$ were found to be the most prevalent risky genes for SDCM. The five significantly risky genes out of the 24 genes, namely MYBPC3, SCN 5A, MYH7, MYPN, and $L D B 3$, had a combined prevalence of $47 \%$ in the patient cohort, which accounted for $78 \%$ of the prevalence of the total risk variants. In addition, 30 novel variants with a potential to increase the risk of DCM were identified in this study.

\section{Comparison of the prevalence of risk variants with other studies}

The prevalence of $61 \%$ for total risk variants observed in this study was higher than that reported in previous studies that included both FDCM and SDCM. In a study by Millat et al., a prevalence of $19 \%$ was observed in 105 DCM patients (8). Another study by Hershberger et al. was $11.5 \%$ in 313 DCM 
patients, although the study cohorts of these studies contained both FDCM and SDCM patients (32). We consider that these differences are partly due to the fact that our study included more genes than these previous studies. For example, only six genes (MYH7, TNNT2, SCN5A, TCAP, $\angle D B 3$, and CSRP3) were screened in the 313-case study, which excluded two genes with high prevalence (MYBPC3 and $M Y P N$ ) found in our study. Given the fact that there are more DCM-associated genes, the actual prevalence of genetic risk variants in SDCM patients would be expected to be even higher. Indeed, a later study from Hershberger et al. including additional genes further expanded the prevalence (up to $27 \%$ ) of the total variants that are likely cause DCM (7). The second factor contributing to this difference is the criteria used to define risk variants. In this study, we used a statistics-based criterion that compares prevalence; a variant was considered to be risky when its prevalence in the DCM cohort was significantly higher than that in the general population (on the condition that the prevalence of this variant in the Chinese population was similar to that in the general population). However, more complicated criteria, which possibly depend on familial cases to a greater extent, were used previously. For example, in the 313-case study, a variant was considered disease-causing if it caused a change in a conserved amino acid, a frameshift, premature truncation, a mis-splicing event, and also segregated with the disease in multiple affected individuals or was identified in multiple unrelated probands, or had previously been reported to be associated with DCM (32). We believe that the criteria used in the present study are more appropriate for detection of novel risk variants, especially in sporadic cases where segregation of multiple occurrence is unlikely. In the risk variants identified by this criterion, $51 \%$ were predicted to be functionally damaging by both the PP2 and SIFT algorithms (in addition to the frame-shift and non-sense variants), and there were significantly more predicted damaging variants in the risky group than in the non-risky group, with a $\mathrm{P}$ value of 0.000 . These facts confirm the effectiveness of the criteria used in our study. With the rapid expansion of population genomic databases, the accuracy of identification of risk variants using statistics-based criteria should be rapidly increased.

Although our study reported a high prevalence of risk variants, the major fraction of the prevalence was attributed to the variants hosted in a few genes. The five risky genes with the highest prevalence (MYBPC3, SCN5A, MYH7, $M Y P N$, and $L D B 3$ ) were distributed in $47 \%$ of the total SDCM patients and $78 \%$ of patients with risk variants. This distribution overlaps with the five genes with the highest prevalence (LMNA, MYBPC3, MYH7, MYH6, and TNNT2) previously obtained from a mixed cohort mainly containing Caucasian patients with FDCM and SDCM. Determination of the genes with the highest prevalence of risky genes may assist in clinical practice by narrowing down the genes to be screened and saving the cost of diagnostic tests. Although the 24 candidates are not the whole targeted genes of SDCM, the results of the study showed the genetic variants from the candidate genes, suggesting the genetic variants are common in DCM even though the patients with no familial history. Besides the environment factors, the genetic factor plays an important role in the pathogenesis of SDCM. The findings of the research indicate the genetic screening is valuable for DCM patients in clinical practice. In addition, our study used next-generation sequencing technology rather than chain termination to determine the DNA sequence, which is faster and more cost-efficient (29). The accurate sequencing results in an array of multiple genes obtained in our study confirm that this highthroughput sequencing technology facilitates diagnostic classification and can improve risk stratification in affected patients.

It is important to note that in this study, the DCM patients were diagnosed as sporadic cases primarily based on the patients' recollection of their family history, which is sometimes inaccurate, and was not verified by medical examination. Therefore, the DCM cases in this study can only be considered as "apparently" sporadic.

\section{Are genetic factors a risk for DCM in sporadic cases?}

The role of genetic factors in the pathogenesis of SDCM remains largely unknown. Previous population studies mainly from Caucasian population showed that the prevalence of disease-causing mutations of some DCMassociated genes is similar for SDCM and FDCM $(7,30)$. Our study conducted in a different ethnic group, namely Chinese, are therefore helpful to further map out the role of genetic factors in SDCM.

Mutational screening studies, such as the present study, provide clear evidence to show that genetic variation has a relation to SDCM. However, compared to the familial cases, it is more difficult to determine the contribution of genetic factors in sporadic cases that do not involve family history, i.e., cases where the disease is not apparently hereditary. Several hypothetical interpretations could be offered. Firstly, it appears that each individual risky variant only 
modestly increases the risk, which is not sufficient to result in the onset of the disease by itself. Therefore, those who carry multiple risk variants are likely to develop the disease. Indeed, 14 of the $40(35 \%)$ patients with risk variants in our study were found to carry multiple risk variants. We cannot exclude the possibility of patients with single risk variants concurrently carrying risk variants of DCM-associated genes, either known or unknown at present, that are not included in our study.

In addition to genetic factors, environmental factors may play a significant role in the expression of pathogenic mechanisms. Our previous study on a segregated DCM family with A1180V of SCN5A (which encodes the cardiac sodium channel) demonstrated that the functional phenotype of this genetic variation was specifically aggravated at high heart rates (26). The results of that study suggested that the risk of DCM of this variation increases in those carriers with physical activity and lifestyles that increase the average daily heart rate. Notably, A1180V of $S C N 5 A$ was found to be a risky variant in the present study.

\section{Is there a specific phenotype that differentiates SDCM patients with risk variants from those without?}

A unique result of this study is the report of summarized symptoms and the statistical comparison of the symptoms between patients with and without risk variants (shown in Tables S2,S3). Our data clearly demonstrated that there were no differences in the profile of DCM-related symptoms between these two patient groups. DCM was the only phenotype for individuals who carried risk variants reported in our study. No other clear genotypephenotype correlations could be concluded from the data. A clear unique phenotype-genotype relationship does not appear to be present for individual genes either. For example, many DCM patients with variants in $L M N A$ and $S C N 5 A$ were reported to suffer from conduction system diseases (26,33); however, in our study cohort, only one patient had first-degree atrioventricular block, whereas other patients carrying risk variants in $L M N A$ and $S C N 5 A$ were not diagnosed with any conduction system diseases. Also, genes listed among the highest prevalence encode proteins (myosin-binding protein $\mathrm{C}$, cardiac sodium channel, $\beta$-myosin heavy chain, myopalladin, and cypher), which have markedly different functions, implicate the heterogeneity of the phenotype-genotype relation. In addition, we noticed that patients carrying multiple variants did not exhibit aggravated symptoms (see Table S2), indicating an absence of additive effects for risk variants on the symptoms of the disease. Certainly, we do not exclude the possibility that some individual variants may not directly cause DCM. However in these cases, DCM is not primary, which is not the concern of this study. Taken together, our data supports the hypothesis that the presence of a risky genotype in SDCM only imposes an increased risk for the onset of DCM, but does not affect the progress of DCM after the onset of the disease. However, the current study does not contain sufficient information regarding the mechanism through which risk variants lead to the onset of SDCM, which was not the purpose of this study and requires further investigation.

\section{Conclusions}

In summary, this study suggests that at-risk genomic variants are a major pathogenic factor of SDCM, and $M Y B P C 3, S C N 5 A, M Y H 7, M Y P N$, and $L D B 3$ are the major genes hosting the at-risk genomic variants. This study also identifies a number of novel variants that are possibly associated with SDCM. These results not only expand the spectrum of DCM genetics, but also provide new information that helps to provide insights into the pathogenesis of SDCM.

\section{Acknowledgments}

Funding: This study was supported by the Surface Project of National Natural Science Foundation of China (82070242 to Lei $\mathrm{Xu}$ ); and the National Science Fund for Distinguished Young Scholars (81725002 to Aijun Sun); and the Innovation Program of Shanghai Municipal Education Commission to Aijun Sun.

\section{Footnote}

Reporting Checklist: The authors have completed the STROBE reporting checklist. Available at https://atm. amegroups.com/article/view/10.21037/atm-21-6774/rc

Data Sharing Statement: Available at https://atm.amegroups. com/article/view/10.21037/atm-21-6774/dss

Conflicts of Interest: All authors have completed the ICMJE uniform disclosure form (available at https://atm. amegroups.com/article/view/10.21037/atm-21-6774/coif). The authors have no conflicts of interest to declare. 
Ethical Statement: The authors are accountable for all aspects of the work in ensuring that questions related to the accuracy or integrity of any part of the work are appropriately investigated and resolved. All procedures performed in this study involving human participants were in accordance with the Declaration of Helsinki (as revised in 2013). This cross-sectional clinical investigation was conducted in compliance with the guidelines for genetic research in the protocol approved by the Ethics Committees of Zhongshan Hospital (No. 2006-87). All participants signed a written informed consent.

Open Access Statement: This is an Open Access article distributed in accordance with the Creative Commons Attribution-NonCommercial-NoDerivs 4.0 International License (CC BY-NC-ND 4.0), which permits the noncommercial replication and distribution of the article with the strict proviso that no changes or edits are made and the original work is properly cited (including links to both the formal publication through the relevant DOI and the license). See: https://creativecommons.org/licenses/by-nc-nd/4.0/.

\section{References}

1. Rosenbaum AN, Agre KE, Pereira NL. Genetics of dilated cardiomyopathy: practical implications for heart failure management. Nat Rev Cardiol 2020;17:286-97.

2. Peters S, Johnson R, Birch S, et al. Familial Dilated Cardiomyopathy. Heart Lung Circ 2020;29:566-74.

3. Xu JH, Gu JY, Guo YH, et al. Prevalence and Spectrum of NKX2-5 Mutations Associated With Sporadic Adult-Onset Dilated Cardiomyopathy. Int Heart J 2017;58:521-9.

4. Asselbergs FW, Sammani A, Elliott P, et al. Differences between familial and sporadic dilated cardiomyopathy: ESC EORP Cardiomyopathy \& Myocarditis registry. ESC Heart Fail 2021;8:95-105.

5. Li J, Liu WD, Yang ZL, et al. Prevalence and spectrum of GATA4 mutations associated with sporadic dilated cardiomyopathy. Gene 2014;548:174-81.

6. Zhou W, Zhao L, Jiang JQ, et al. A novel TBX5 lossof-function mutation associated with sporadic dilated cardiomyopathy. Int J Mol Med 2015;36:282-8.

7. Hershberger RE, Norton N, Morales A, et al. Coding sequence rare variants identified in MYBPC3, MYH6, TPM1, TNNC1, and TNNI3 from 312 patients with familial or idiopathic dilated cardiomyopathy. Circ Cardiovasc Genet 2010;3:155-61.
8. Millat G, Bouvagnet P, Chevalier P, et al. Clinical and mutational spectrum in a cohort of 105 unrelated patients with dilated cardiomyopathy. Eur J Med Genet 2011;54:e570-5.

9. Li D, Morales A, Gonzalez-Quintana J, et al. Identification of novel mutations in RBM20 in patients with dilated cardiomyopathy. Clin Transl Sci 2010;3:90-7.

10. Li M, Xia S, Xu L, et al. Genetic analysis using targeted next-generation sequencing of sporadic Chinese patients with idiopathic dilated cardiomyopathy. J Transl Med 2021;19:189.

11. Hershberger RE, Morales A, Siegfried JD. Clinical and genetic issues in dilated cardiomyopathy: a review for genetics professionals. Genet Med 2010;12:655-67.

12. Hershberger RE, Siegfried JD. Update 2011: clinical and genetic issues in familial dilated cardiomyopathy. J Am Coll Cardiol 2011;57:1641-9.

13. Bozkurt B, Colvin M, Cook J, et al. Current Diagnostic and Treatment Strategies for Specific Dilated Cardiomyopathies: A Scientific Statement From the American Heart Association. Circulation 2016;134:e579-646.

14. Pinto YM, Elliott PM, Arbustini E, et al. Proposal for a revised definition of dilated cardiomyopathy, hypokinetic non-dilated cardiomyopathy, and its implications for clinical practice: a position statement of the ESC working group on myocardial and pericardial diseases. Eur Heart J 2016;37:1850-8.

15. Xing Y, Ichida F, Matsuoka T, et al. Genetic analysis in patients with left ventricular noncompaction and evidence for genetic heterogeneity. Mol Genet Metab 2006;88:71-7.

16. Van Driest SL, Vasile VC, Ommen SR, et al. Myosin binding protein $\mathrm{C}$ mutations and compound heterozygosity in hypertrophic cardiomyopathy. J Am Coll Cardiol 2004;44:1903-10.

17. Bahrudin U, Morisaki H, Morisaki T, et al. Ubiquitinproteasome system impairment caused by a missense cardiac myosin-binding protein C mutation and associated with cardiac dysfunction in hypertrophic cardiomyopathy. J Mol Biol 2008;384:896-907.

18. Song L, Zou Y, Wang J, et al. Mutations profile in Chinese patients with hypertrophic cardiomyopathy. Clin Chim Acta 2005;351:209-16.

19. Lin J, Zheng DD, Tao Q, et al. Two novel mutations of the MYBPC3 gene identified in Chinese families with hypertrophic cardiomyopathy. Can J Cardiol 2010;26:518-22.

20. Tajsharghi H, Oldfors A. Myosinopathies: pathology and mechanisms. Acta Neuropathol 2013;125:3-18. 
21. Alcalai R, Seidman JG, Seidman CE. Genetic basis of hypertrophic cardiomyopathy: from bench to the clinics. J Cardiovasc Electrophysiol 2008;19:104-10.

22. Laredo R, Monserrat L, Hermida-Prieto M, et al. Beta-myosin heavy-chain gene mutations in patients with hypertrophic cardiomyopathy. Rev Esp Cardiol 2006;59:1008-18.

23. Dellefave LM, Pytel P, Mewborn S, et al. Sarcomere mutations in cardiomyopathy with left ventricular hypertrabeculation. Circ Cardiovasc Genet 2009;2:442-9.

24. Viswanathan PC, Benson DW, Balser JR. A common SCN5A polymorphism modulates the biophysical effects of an SCN5A mutation. J Clin Invest 2003;111:341-6.

25. Chiu SN, Wu MH, Su MJ, et al. Coexisting mutations/ polymorphisms of the long QT syndrome genes in patients with repaired Tetralogy of Fallot are associated with the risks of life-threatening events. Hum Genet 2012;131:1295-304.

26. Ge J, Sun A, Paajanen V, et al. Molecular and clinical characterization of a novel SCN5A mutation associated with atrioventricular block and dilated cardiomyopathy. Circ Arrhythm Electrophysiol 2008;1:83-92.

27. Hwang HW, Chen JJ, Lin YJ, et al. R1193Q of SCN5A, a Brugada and long QT mutation, is a common polymorphism in Han Chinese. J Med Genet 2005;42:e7; author reply e8.

Cite this article as: Shen C, Xu L, Sun X, Sun A, Ge J. Genetic variants in Chinese patients with sporadic dilated cardiomyopathy: a cross-sectional study. Ann Transl Med 2022;10(3):129. doi: 10.21037/atm-21-6774
28. Jáchymová M, Muravská A, Paleček T, et al. Genetic variation screening of TNNT2 gene in a cohort of patients with hypertrophic and dilated cardiomyopathy. Physiol Res 2012;61:169-75.

29. Gu W, Miller S, Chiu CY. Clinical Metagenomic NextGeneration Sequencing for Pathogen Detection. Annu Rev Pathol 2019;14:319-38.

30. Kwon HW, Lee SY, Kwon BS, et al. Long QT syndrome and dilated cardiomyopathy with SCN5A p.R1193Q polymorphism: cardioverter-defibrillator implantation at 27 months. Pacing Clin Electrophysiol 2012;35:e243-6.

31. Lakdawala NK, Funke BH, Baxter S, et al. Genetic testing for dilated cardiomyopathy in clinical practice. J Card Fail 2012;18:296-303.

32. Hershberger RE, Parks SB, Kushner JD, et al. Coding sequence mutations identified in MYH7, TNNT2, SCN5A, CSRP3, LBD3, and TCAP from 313 patients with familial or idiopathic dilated cardiomyopathy. Clin Transl Sci 2008;1:21-6.

33. Kawakami H, Ogimoto A, Tokunaga N, et al. A Novel Truncating LMNA Mutation in Patients with Cardiac Conduction Disorders and Dilated Cardiomyopathy. Int Heart J 2018;59:531-41.

(English Language Editor: A. Kassem) 


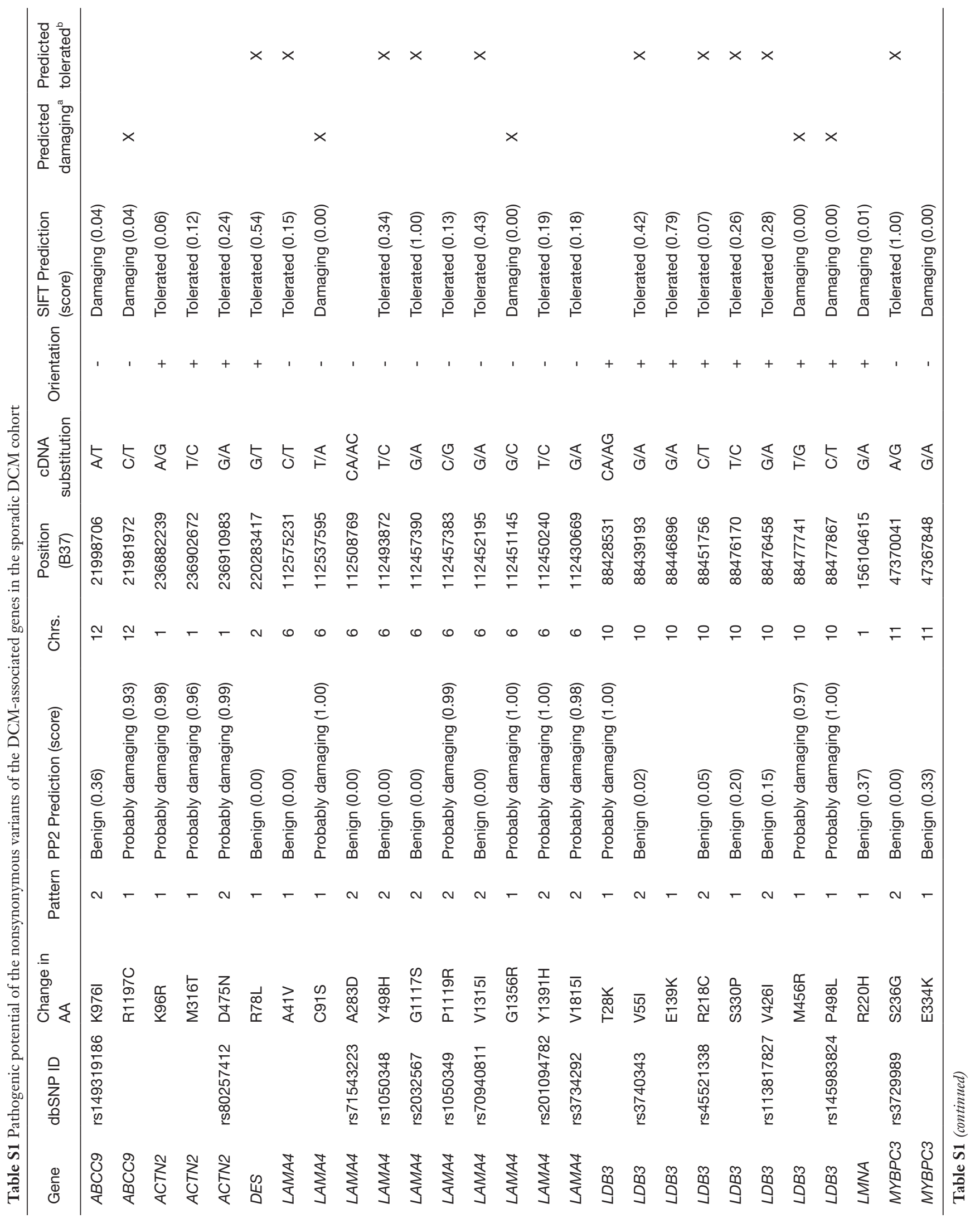




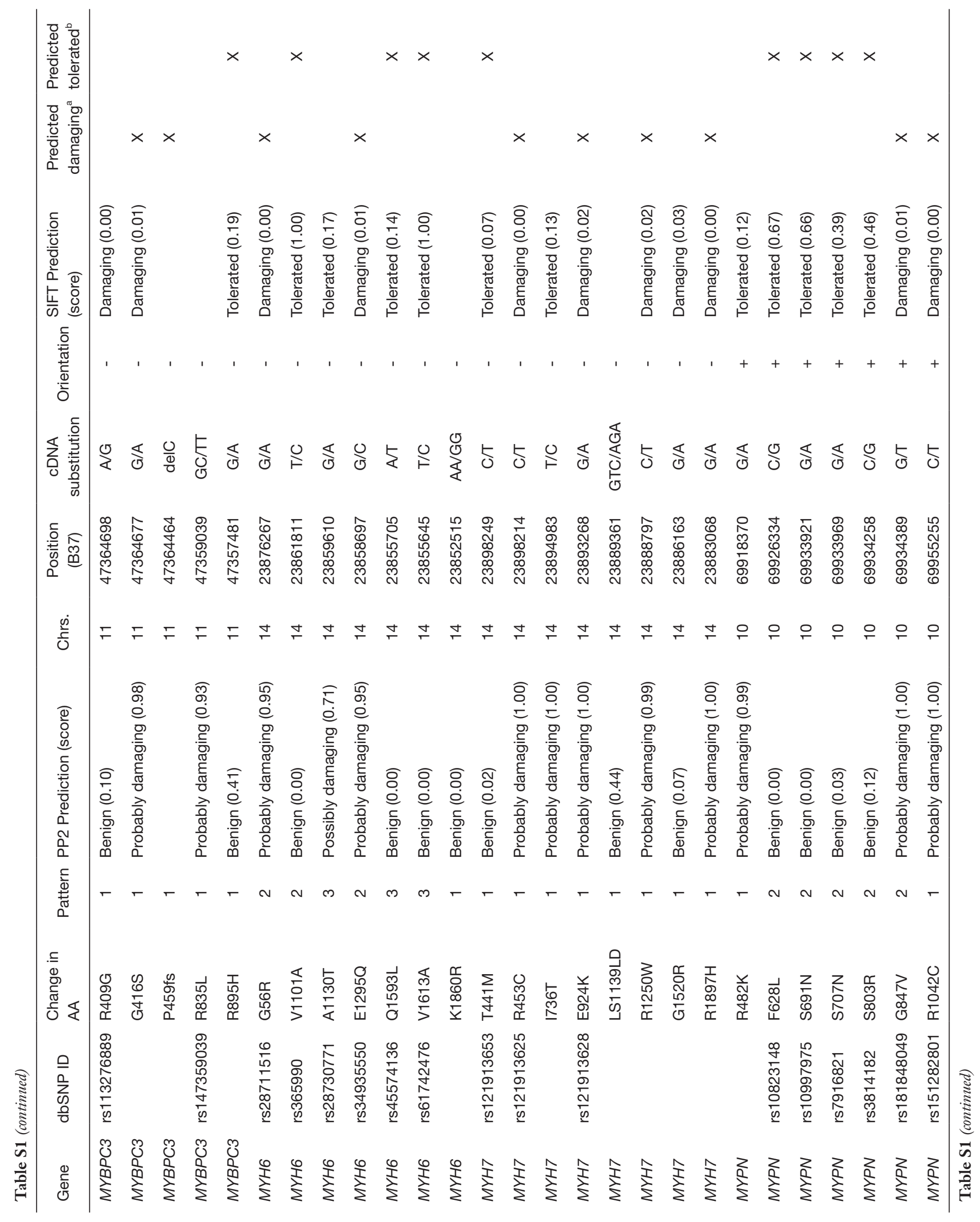




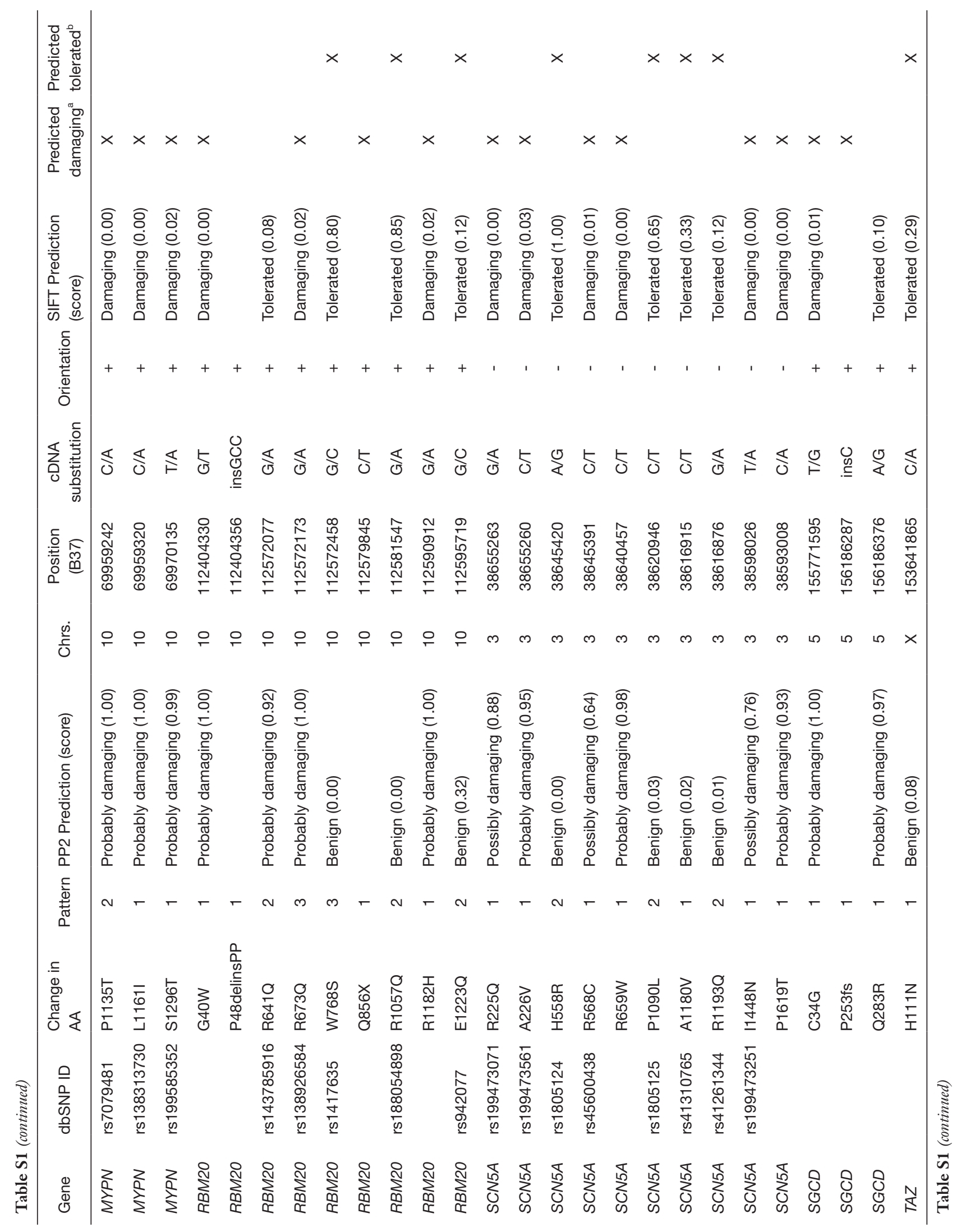




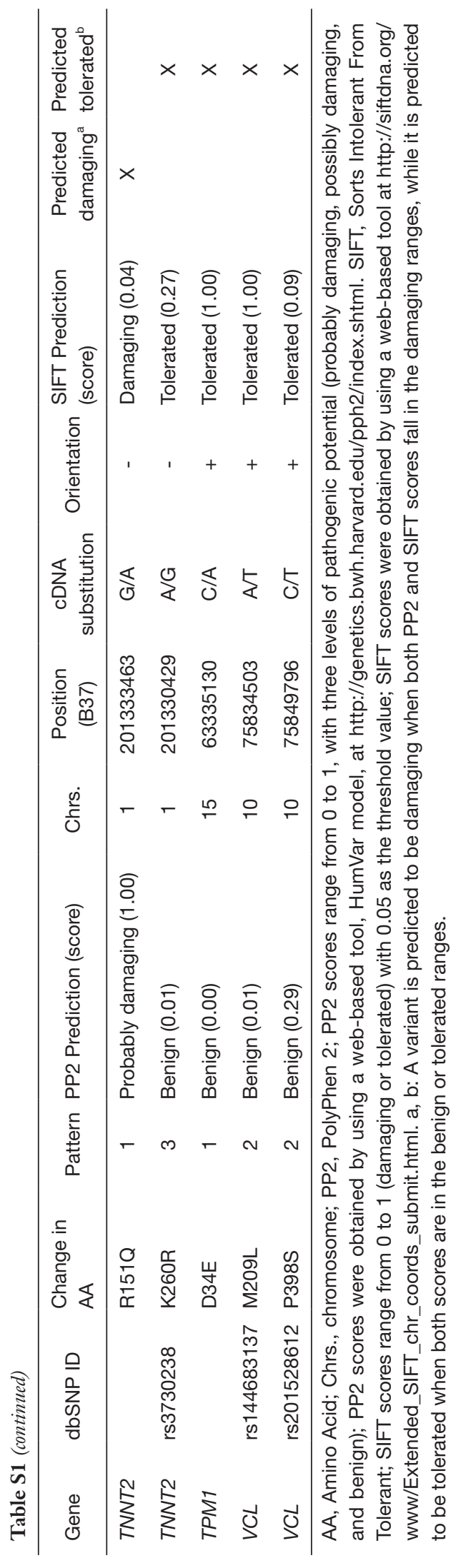


Table S2 Comparison of symptoms between the sporadic DCM patients with and without risk variants

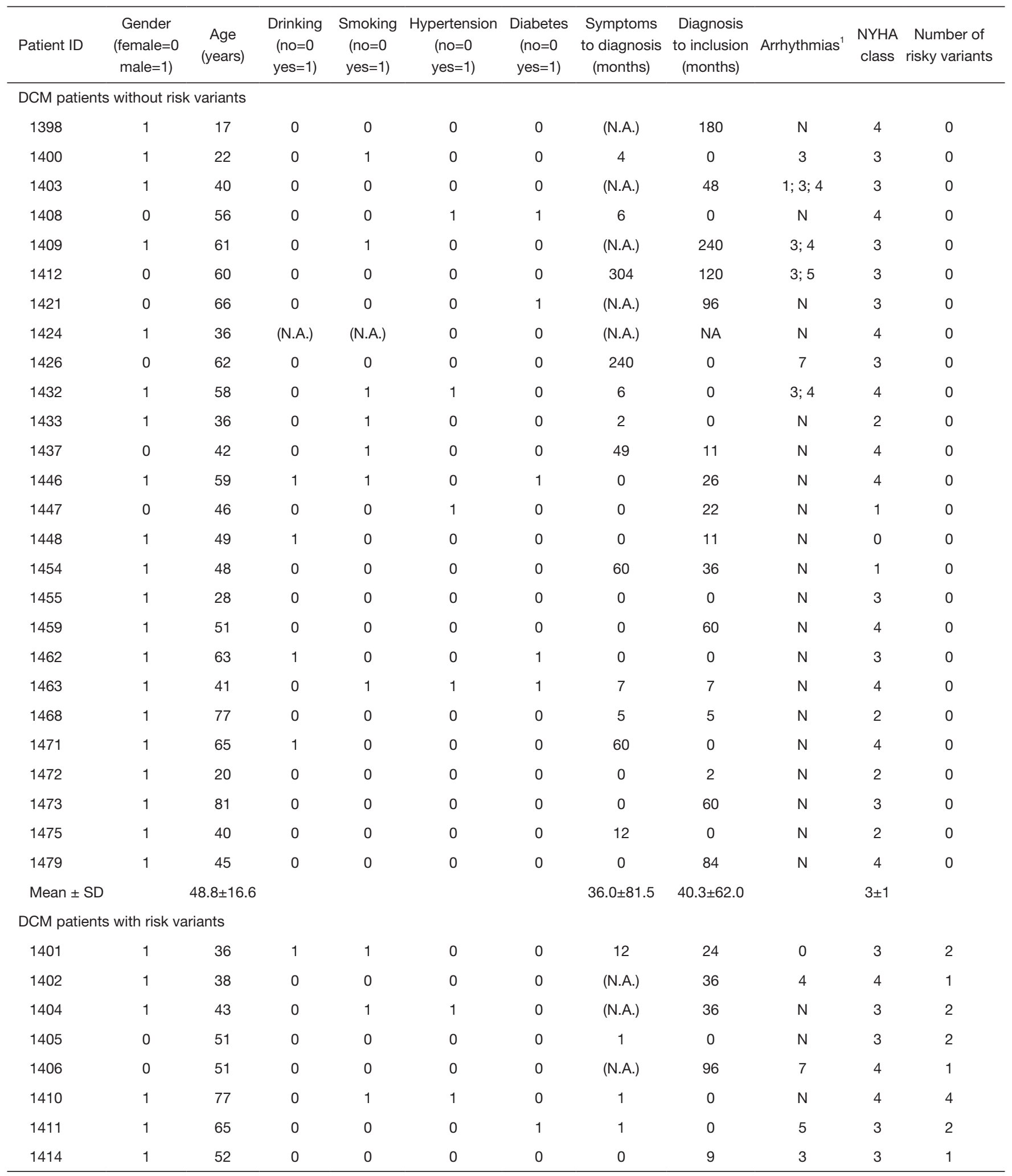

Table S2 (continued) 
Table S2 (continued)

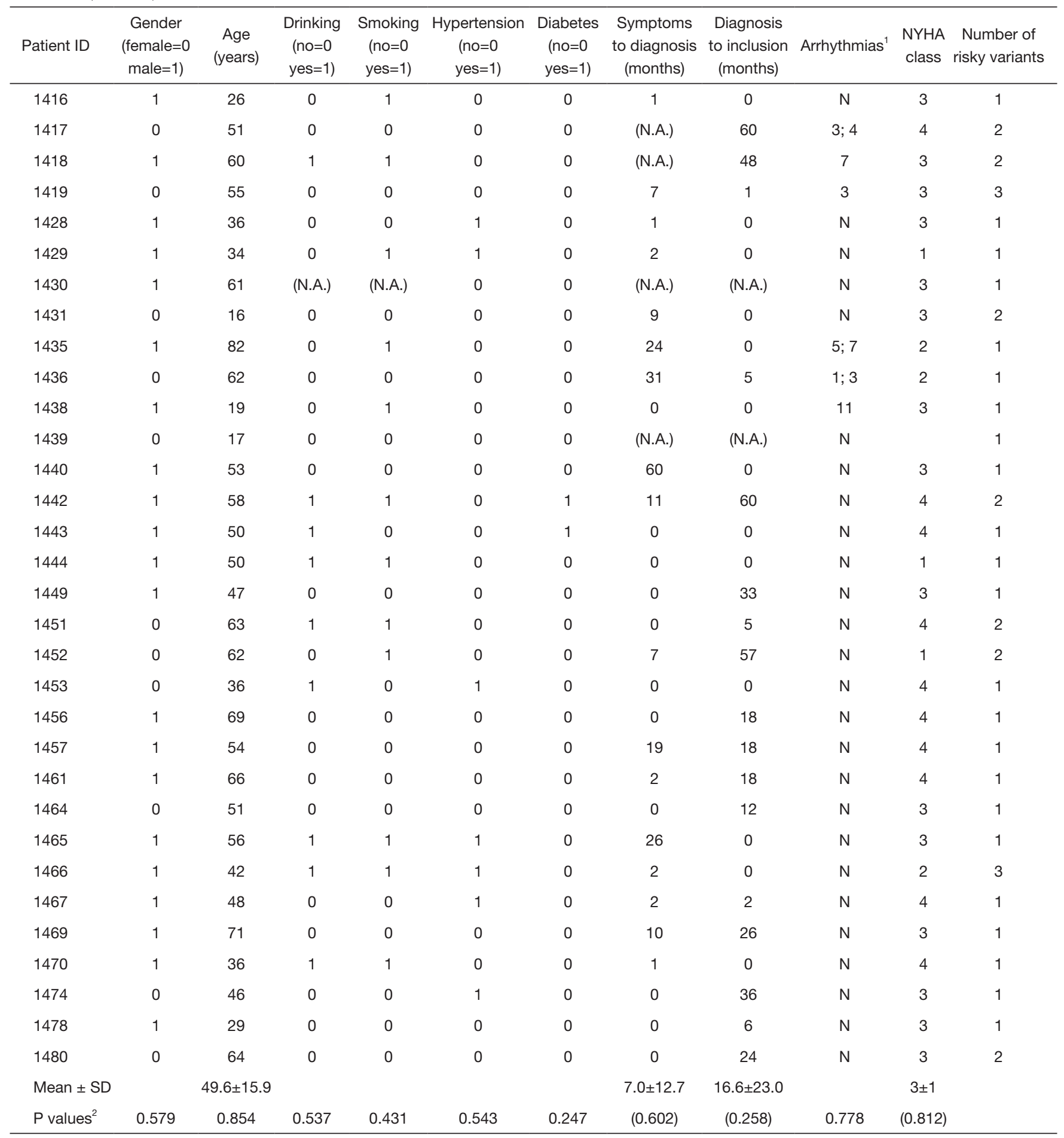

1: Arrhythmias: atrial fibrillation or flutter=0; atrial premature beat=1; atrial tachycardia=2; ventricular premature beat=3; ventricular tachycardia=4; first-degree AV block=5; third-degree AV block=6; complete left or right bundle branch block=7. 2: Compared with the corresponding parameter of the patients without risky variants. $\mathrm{P}$ values in the parentheses were obtained from the Mann-Whitney rank sum test. N, no symptom; N.A., data not available; SD, standard deviation. 
Table S3 Comparison of echocardiographic parameters between the sporadic DCM patients with and without risk variants

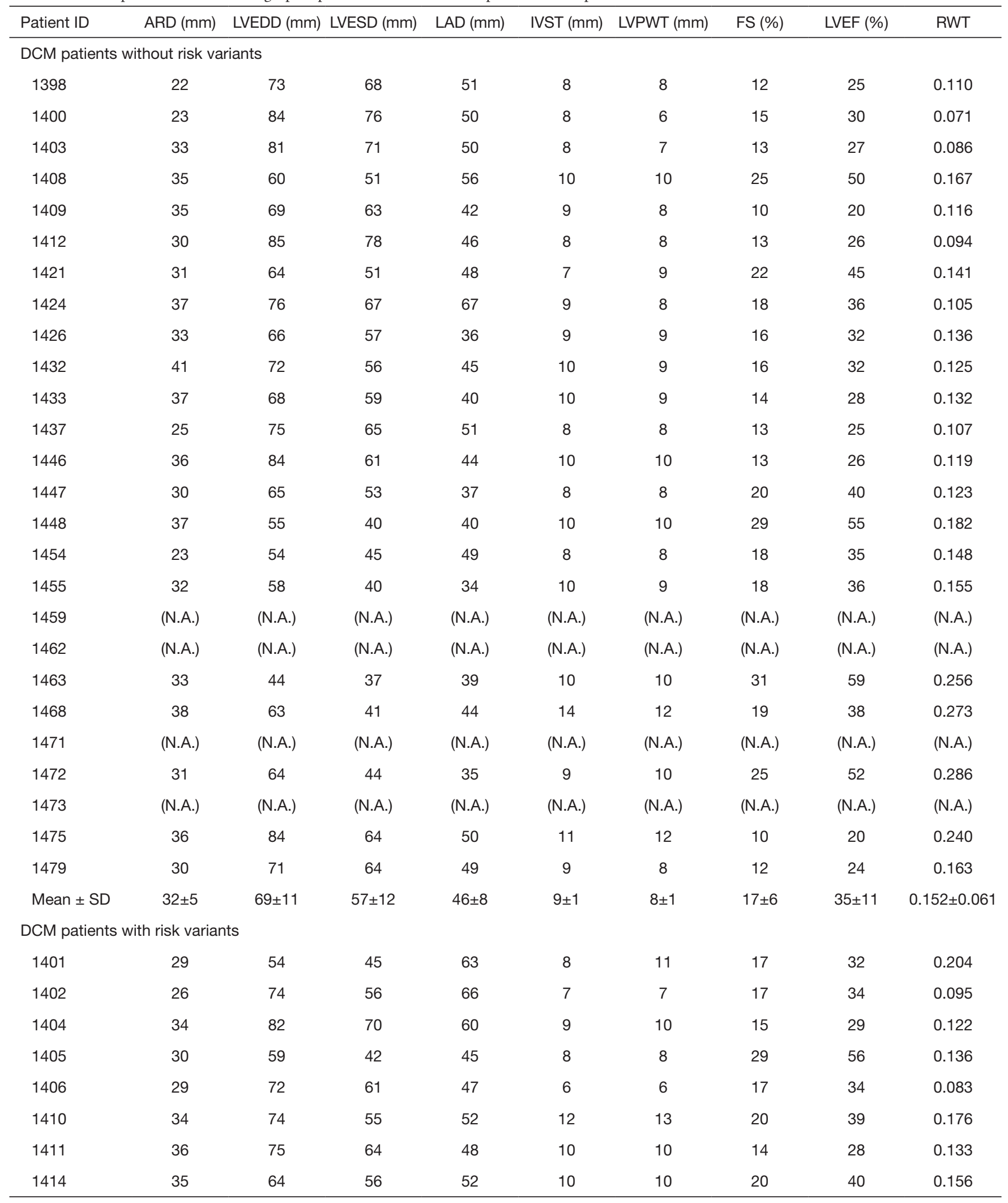

Table S3 (continued) 
Table S3 (continued)

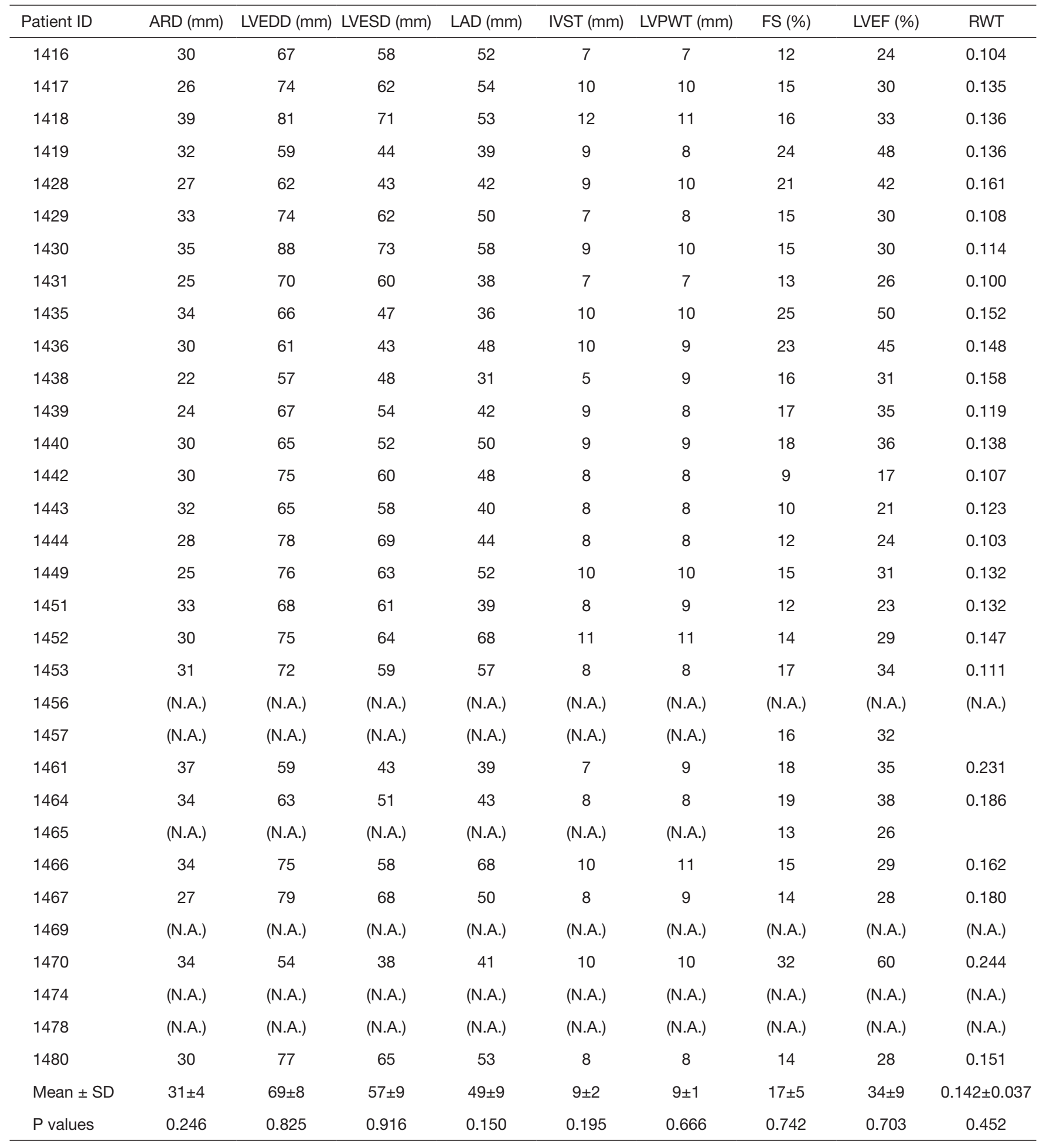

Compared with the corresponding parameter of the patients without risk variants; SD, standard deviation. ARD, aortic root diameter; FS, shortening fraction; LAD, left atrial diameter; LVEDD, left ventricular end-diastolic diameter; LVEF, left ventricular ejection fraction; LVESD, left ventricular end-systolic diameter; LVPWT, left ventricule post wall thickness; IVST, interventricular septal thickness; N, no symptom; N.A., data not available; RWT, relative wall thickness. 